\title{
The Influence of Elastic Anisotropy on the X-ray Topographic Image Width of Pure Screw Dislocations
}

\author{
BY H. KLAPPER \\ Institut für Kristallographie der Technischen Hochschule Aachen, 51 Aachen, Germany (BRD)
}

(Received 29 April 1974)

\begin{abstract}
The X-ray topographic image widths of pure screw dislocation lines along a twofold axis of orthorhombic ammonium hydrogen oxalate hemihydrate, lithium formate monohydrate, thiourea, and monoclinic potassium hydrogen oxalate have been studied. The widths observed on topographs made with imaging planes perpendicular to the dislocation line vary with the orientation of the incidence plane. This effect results from the elastic anisotropy, which gives rise to a varying slope of the helical surface formed by the distorted lattice planes. The displacements, strains, and effective misorientations of the imaging planes are quantitatively analysed for screw dislocations along a twofold axis or perpendicular to a mirror plane, taking into account elastic anisotropy. The image widths for various orientations of the incidence plane are calculated. The ratio of maximum and minimum widths is $W_{\max } / W_{\min }=c_{44} / c_{55}$, with $c_{44}$ and $c_{55}$ the maximum and minimum shear resistances, respectively, in the plane perpendicular to the dislocation line. Observed and calculated ratios are in good agreement.
\end{abstract}

J. Appl. Cryst. (1975). 8, 204

\section{Electron-Diffraction and Electron-Microscopy Methods for the Study of Ordering in Crystals}

\author{
BY J.M.COWLEY \\ Department of Physics, Arizona State University, Tempe, Arizona 85281, U.S.A.
}

(Received 11 June 1974)

\begin{abstract}
The diffraction of electrons is, in principle, a very sensitive probe of the relative atomic positions in ordered or disordered crystals. The full potential of electron diffraction and electron microscopy methods for the study of local atomic arrangements has not been realized because of the difficulties of conducting experiments under sufficiently well defined conditions and because of the complications introduced by the dynamical diffraction processes involved. Even crude approximations, if physically sound, can provide a great deal of information not accessible by other methods. With increased understanding of the nature of the dynamical scattering processes, the techniques are now being made even more powerful and the range of applications is becoming even greater. Single-crystal diffraction patterns have long been used as a convenient, rapid method for the detection and study of the diffuse scattering due to crystal defects and the weak additional spots due to superlattice formation. The possibility of obtaining single-crystal patterns from extremely small volumes of material gives an immediate advantage over X-ray diffraction techniques for which suitable single crystal samples are of ten difficult or impossible to prepare, as in the case of the Ti-O phases studied by Castles, J. C., Cowley, J. M. \& Spargo A. E. C. [Acta Cryst. (1971). A 27, 376-383]. It cannot be assumed, however, that diffuse scattering in electron diffraction patterns can be interpreted by simple kinematical theory. The presence of Kikuchi lines in the diffuse scattering is an immediate indication of dynamical scattering effects. More seriously, the dynamical interactions through the Bragg reflections can modify the whole configuration of diffuse scattering. The experimental and computational results of Fisher, P. M. J. [Proc. Int. Conf. Electron Diffraction and Crystal Defects, Melbourne (1965), Paper I H-4] showed for example that strong dynamical interactions through Bragg reflections can remove the size-effect asymmetry of the short-range-order diffuse peaks given by disordered, thin copper-gold crystals. Such effects can be described in terms of a tendency for the regular array of strong Bragg reflections to impose its own symmetry on the diffuse scattering distribution. Methods for the quantitative evaluation of dynamical diffraction effects on diffuse scattering have been developed by Gjønnes and collaborators and have been used very effectively, for example, in the analysis of diffuse scattering patterns given by defects in vanadium oxides [Andersson, B., Gjønnes, J. \& Tafto, J. [Acta Cryst. (1974). A 30, 216-224]. There is one class of specimen in which the dynamical effects in the diffuse scattering can be separated out relatively simply. This is the case of thin crystalline
\end{abstract}

